\title{
Checklist of the family Sphaeroceridae (Diptera) of Finland
}

\author{
Antti Haarto', Jere Kahanpää ${ }^{2}$ \\ I Zoological Museum, Section of Biodiversity and Environmental Science, Department of Biology, FI-20014 \\ University of Turku, Finland 2 Finnish Museum of Natural History, Zoology Unit, P.O. Box 17, FI-00014 \\ University of Helsinki, Finland \\ Corresponding author: Antti Haarto (ahaarto@gmail.com) \\ Academic editor: J. Salmela | Received 12 February 2014 | Accepted 23 March 2014 | Published 19 September 2014 \\ http://zoobank.org/D3D5D02A-BB03-4168-881F-98740C81AD0A \\ Citation: Haarto A, Kahanpää J (2014) Checklist of the family Sphaeroceridae (Diptera) of Finland. In: Kahanpää J, \\ Salmela J (Eds) Checklist of the Diptera of Finland. ZooKeys 441: 325-332. doi: 10.3897/zookeys.441.7250
}

\begin{abstract}
A checklist of the family Sphaeroceridae (Diptera) recorded from Finland.
\end{abstract}

\section{Keywords}

Checklist, Finland, Diptera, Sphaeroceridae

\section{Introduction}

Sphaeroceridae or lesser dung flies are mostly small and dull dark brown to grey acalyptrate flies. They are easily distinguished from other acalyptratae because of the short and thickened basal tarsomeres of hind legs. Larvae of Sphaeroceridae develop in decaying organic matter such as decaying vegetation, dung and rotten fungi.

The family has gathered little interest among entomologists in Finland. Walter Hackman concentrated on the taxonomy of the subfamily Copromyzinae (Hackman 1965) and investigated the dipterous fauna, including sphaerocerids, in the burrows of small mammals (Hackman 1963). Hackman (1980) published the latest list of the Finnish sphaerocerid species. Further species were added in several revisions of Eu- 
ropean taxa by Roháček $(1982,1983,1991)$. There has been increasing interest in sphaerocerids in Finland after the year 2000 (see Haarto and Kahanpää 2013).

The classification follows the world catalogue of Sphaeroceridae (Roháček et al. 2001, Marshall et al. 2011).

\section{Number of species:}

World: 1571 species (Pape et al. 2011)

Europe: 257 species

Finland: 118 species

Faunistic knowledge level in Finland: average

\section{Checklist}

suborder Brachycera Macquart, 1834

clade Eremoneura Lameere, 1906

clade Cyclorrhapha Brauer, 1863

infraorder Schizophora Becher, 1882

clade Muscaria Enderlein, 1936

parvorder Acalyptratae Macquart, 1835

superfamily Sphaeroceroidea Macquart, 1835

SPHAEROCERIDAE Macquart, 1835

COPROMYZINAE Stenhammar, 1854

ALLOBORBORUS Duda, 1923

Alloborborus pallifrons (Fallén, 1820)

= flavipennis (Haliday, 1836)

BORBORILLUS Duda, 1923

Borborillus uncinatus (Duda, 1923)

Borborillus vitripennis (Meigen, 1830)

= longipennis (Haliday, 1836)

COPROMYZA Fallén, 1810

Copromyza borealis Zetterstedt, 1847

Copromyza equina Fallén, 1820

Copromyza nigrina (Gimmerthal, 1847)

= similis (Collin, 1930)

Copromyza stercoraria (Meigen, 1830)

CRUMOMYIA Macquart, 1835

= Apterina Macquart, 1835

Crumomyia fimetaria (Meigen, 1830)

Crumomyia gelida Hackman, 1965

Crumomyia glabrifrons (Meigen, 1830)

Crumomyia nigra (Meigen, 1830) 
Crumomyia nitida (Meigen, 1830)

Crumomyia notabilis (Collin, 1902)

$=$ glacialis misid.

Crumomyia pedestris (Meigen, 1830

Crumomyia pruinosa (Richards, 1932)

$=$ annulus misid.

= rufoannulata misid.

Crumomyia setitibialis (Spuler, 1925)

$=$ freyi $($ Hackman, 1965)

$=$ annulipes misid.

$=$ roseri misid.

LOTOPHILA Lioy, 1864

Lotophila atra (Meigen, 1830)

NORRBOMIA Papp, 1988

Norrbomia costalis (Zetterstedt, 1847)

Norrbomia fumipennis (Stenhammar, 1855)

Norrbomia hispanica (Duda, 1923)

Norrbomia sordida (Zetterstedt, 1847)

SPHAEROCERINAE Macquart, 1835

ISCHIOLEPTA Lioy, 1864

Ischiolepta crenata (Meigen, 1838)

Ischiolepta denticulata (Meigen, 1830)

= paracrenata (Duda, 1920)

Ischiolepta micropyga (Duda, 1938)

Ischiolepta nitida (Duda, 1920)

= denticulata auct. nec (Meigen, 1830)

Ischiolepta pusilla (Fallén, 1820)

Ischiolepta scabricula (Haliday, 1836)

Ischiolepta vaporariorum (Haliday, 1836)

LOTOBIA Lioy, 1864

Lotobia pallidiventris (Meigen, 1830)

SPHAEROCERA Latreille, 1804

= Cypsela Meigen, 1800 suppr.

Sphaerocera curvipes Latreille, 1805

Sphaerocera monilis Haliday, 1836

LIMOSININAE Frey, 1921

APTEROMYIA Vimmer, 1929

Apteromyia claviventris (Strobl, 1909)

BIFRONSINA Roháček, 1983

Bifronsina bifrons (Stenhammar, 1855)

CHAETOPODELLA Duda, 1920

sg. Chaetopodella Duda, 1920

Chaetopodella scutellaris (Haliday, 1836) 
COPROICA Rondani, 1861

Coproica acutangula (Zetterstedt, 1847)

Coproica ferruginata (Stenhammar, 1855)

Coproica hirticula Collin, 1965

Coproica hirtula (Rondani, 1880)

Coproica lugubris (Haliday, 1835)

Coproica pusio (Zetterstedt, 1847)

= pseudolugubris (Duda, 1924)

Coproica vagans (Haliday, 1833)

ELACHISOMA Rondani, 1880

Elachisoma aterrimum (Haliday, 1833)

EULIMOSINA Roháček, 1983

Eulimosina ochripes (Meigen, 1830)

GIGALIMOSINA Roháček, 1983

Gigalimosina flaviceps Zetterstedt, 1847

GONIONEURA Rondani, 1880

= Halidayina Duda, 1918

Gonioneura spinipennis (Haliday, 1836)

HERNIOSINA Roháček, 1983

Herniosina bequaerti (Villeneuve, 1917)

LEPTOCERA Olivier, 1813

= Paracollinella Duda, 1924

Leptocera caenosa (Rondani, 1880)

Leptocera finalis (Collin, 1956)

Leptocera fontinalis (Fallén, 1826)

Leptocera nigra Olivier, 1813

LIMOSINA Macquart, 1835

Limosina silvatica (Meigen, 1830)

MINILIMOSINA Roháček, 1983

sg. Minilimosina Roháček, 1983

Minilimosina baculum Marshall, 1985

Minilimosina bicuspis Roháček, 1993

$=$ trogeri misid.

Minilimosina fungicola (Haliday, 1836)

= exigua (Rondani, 1880)

Minilimosina parvula Stenhammar, 1855

Minilimosina tenera Roháček, 1983

sg. Svarciella Roháček, 1983

Minilimosina guestphalica (Duda, 1918) $=v$-atrum misid.

Minilimosina v-atrum (Villeneuve, 1917) = splendens (Duda, 1928)

Minilimosina vitripennis (Zetterstedt, 1847) 
OPACIFRONS Duda, 1918

Opacifrons coxata (Stenhammar, 1855)

OPALIMOSINA Roháček, 1983

sg. Dentilimosina Roháček, 1983

Opalimosina denticulata (Duda, 1924)

sg. Opalimosina Roháček, 1983

Opalimosina collini (Richards, 1929)

Opalimosina mirabilis (Collin, 1902)

Opalimosina simplex Richards 1929

sg. Pappiella Roháček, 1983

Opalimosina liliputana (Rondani, 1880)

= appendiculata Villeneuve, 1918

PARALIMOSINA Papp, 1973

Paralimosina kaszabi Papp, 1973

PHILOCOPRELLA Richards, 1929

Philocoprella quadrispina (Laurence, 1952)

PHTHITIA Enderlein, 1938

sg. Alimosina Roháček, 1983

Phthitia empirica (Hutton, 1901)

= cadaverina (Duda, 1918)

sg. Collimosina Roháček, 1983

Phthitia spinosa Collin, 1930

sg. Kimosina Roháček, 1983

Phthitia longisetosa (Dahl, 1909)

Phthitia plumosula (Rondani, 1880)

PSEUDOCOLLINELLA Duda, 1924

Pseudocollinella flavilabris (Hackman, 1968)

Pseudocollinella humida (Haliday, 1836)

Pseudocollinella septentrionalis (Stenhammar, 1855)

PTEREMIS Rondani, 1856

Pteremis fenestralis (Fallén, 1820)

= nivalis (Haliday, 1833)

= subaptera Frey, 1946

PULLIMOSINA Roháček, 1983

sg. Dablimosina Roháček, 1983

Pullimosina dabli (Duda, 1918)

sg. Pullimosina Roháček, 1983

Pullimosina heteroneura (Haliday, 1836)

Pullimosina meijerei (Duda, 1918)

Pullimosina moesta (Villeneuve, 1918)

= antennata (Duda, 1918)

Pullimosina pullula (Zetterstedt, 1847)

Pullimosina vulgesta Roháček, 2001 
$=$ moesta auct. nec (Villeneuve, 1918)

RACHISPODA Lioy, 1864

Rachispoda anceps (Stenhammar, 1855)

Rachispoda breviceps (Stenhammar, 1855)

Rachispoda fuscipennis (Haliday, 1833)

Rachispoda hostica Villeneuve, 1917

Rachispoda intermedia (Duda, 1918)

Rachispoda limosa (Zetterstedt, 1820)

Rachispoda lugubrina (Zetterstedt, 1847)

Rachispoda lutosa (Stenhammar, 1855)

= palustris (Collin, 1930)

Rachispoda lutosoidea (Duda, 1938)

= lutosa auct. nec (Stenhammar, 1855)

SPELOBIA Spuler, 1924

Spelobia belanica Roháček, 1983

Spelobia cambrica (Richards, 1929)

Spelobia clunipes (Meigen, 1830)

= crassimana (Haliday, 1836)

Spelobia ibrida Roháček, 1983

Spelobia luteilabris (Rondani, 1880)

Spelobia manicata (Richards, 1927)

Spelobia nana (Rondani, 1880)

Spelobia palmata (Richards, 1927)

Spelobia pappi Roháček, 1983

Spelobia parapusio (Dahl, 1909)

Spelobia pseudonivalis (Dahl, 1909)

Spelobia pseudosetaria (Duda, 1918)

= penetralis (Collin, 1925)

Spelobia rufilabris (Stenhammar, 1855)

Spelobia talparum (Richards, 1927)

Spelobia ulla Roháček, 1983

SPINILIMOSINA Roháček, 1983

Spinilimosina brevicostata (Duda, 1918)

TELOMERINA Roháček, 1983

Telomerina eburnea Roháček, 1983

Telomerina flavipes (Meigen, 1830)

Telomerina pseudoleucoptera (Duda, 1924)

TERRILIMOSINA Roháček, 1983

Terrilimosina racovitzai (Bezzi, 1911)

Terrilimosina schmitzi (Duda, 1918)

THORACOCHAETA Duda, 1918

Thoracochaeta brachystoma (Stenhammar, 1855)

Thoracochaeta zosterae (Haliday, 1833) 
TRACHYOPELLA Duda, 1918

sg. Nudopella Roháček \& Marshall, 1986

Trachyopella leucoptera (Haliday, 1836)

sg. Trachyopella Duda, 1918

Trachyopella atomus (Rondani, 1880)

Trachyopella bovilla Collin, 1954

= coprina misid.

Trachyopella lineafrons (Spuler, 1925)

$=$ atomus misid.

Trachyopella melania (Haliday, 1836)

= villeneuvii (Duda, 1924)

\section{Excluded species}

Rachispoda cilifera (Rondani, 1880) not found within present borders

Minilimosina unica (Papp, 1973) not found within present borders

= hackmani (Roháček, 1977)

\section{Notes}

Spelobia manicata (Richards, 1927) is considered a valid species although Pitkin (1988) synonymized it with Spelobia clunipes (Meigen, 1830).

\section{References}

Haarto A, Kahanpää J (2013) Notes of Finnish Sphaeroceridae (Diptera) with description of the female of Minilimosina tenera Rohacek, 1983. Entomologica Fennica 24: 228-233. http://ojs.tsv.fi/index.php/entomolfennica/article/download/9385/6695

Hackman W (1963) Studies on the dipterous fauna in burrows of voles (Microtus, Clethriono$m y s$ ) in Finland. Acta Zoologica Fennica 102: 1-64.

Hackman W (1965) On the genus Copromyza Fall. (Dipt., Sphaeroceridae), with special reference to the Finnish species. Notulae Entomologicae 45: 33-46.

Hackman W (1980) A Check List of the Finnish Diptera. Notulae entomologicae 60: 17-48, $117-162$.

Marshall SA, Roháček J, Dong H, Buck M (2011) The state of Sphaeroceridae (Diptera: Acalyptratae): a world catalog update covering the years 2000-2010, with new generic synonymy, new combinations, and new distributions. Acta Entomologica Musei Nationalis Pragae 51(1): 217-298. http://www.aemnp.eu/PDF/51_1/51_1_217.pdf

Pape T, Blagoderov V, Mostovski MB (2011) Order Diptera Linnaeus, 1758. In: Zhang Z-Q (Ed) Animal biodiversity: An outline of higher-level classification and survey of 
taxonomic richness. Zootaxa 3148: 222-229. http://www.mapress.com/zootaxa/2011/f/ zt03148p229.pdf

Pitkin BR (1988) Lesser dung flies, Diptera: Sphaeroceridae. Handbooks for the Identification of British Insects, Vol 10, Part 5e. Royal Entomological Society of London, London, 175 pp. Roháček J (1982) Revision of the subgenus Leptocera (s.str.) of Europe. Entomologische Abhandlungen Staatliches Museum für Tierkunde in Dresden 46(1): 1-44.

Roháček J (1983) A monograph and re-classification of the previous genus Limosina Macquart (Diptera, Sphaeroceridae) of Europe. Part II. Beiträge zur Entomologie, Berlin 33: 3-195. Roháček J (1991) A monograph of Leptocera (Rachispoda Lioy) of the West Palaearctic area (Diptera, Sphaeroceridae). Časopis Slezského zemského Muzea, Opava (A) 40: 97-288. Roháček J, Marshall SA, Norrbom AL, Buck M, Quiros DI, Smith I (2001) World Catalog of Sphaeroceridae. Slezské Zemské Muzeum, Opava, 300 pp. ISBN 808622421X. http:// www.uoguelph.ca/debu/catalog.htm 